\section{Review: Psychosocial and psychological interventions reduce postpartum depressive symptoms}

\section{QUESTION}

Question: How effective are psychosocial and psychological interventions compared with usual care for treating postpartum depression?

Outcomes: Primary outcomes were recovery and reduction in depressive symptoms, as defined and measured in the individual trials.

\section{METHODS}

Design: Systematic review with meta-analysis.

Data sources: Cochrane Collaboration Depression, Anxiety, and Neurosis Controlled Trials registers (searched August 2007), the Cochrane Pregnancy and Childbirth Review Group trials register, CENTRAL, MEDLINE (1966-2006), EMBASE (1980-2006), CINAHL (1982-2006), and hand searches of reference lists, and contact with experts in the field.

Study selection and analysis: Randomised controlled trials comparing psychosocial or psychological interventions with usual care for reducing symptoms of postpartum depression were included. Women could be identified using self report measures or by diagnostic interview during the 12 months after delivery. Usual care consisted of any appropriate medical care, including antidepressants or other pharmacotherapy. Published, unpublished, and ongoing trials were included. Two researchers evaluated methodological quality of the trials and extracted data. Authors were contacted for missing data if necessary. Heterogeneity was investigated using the $\mathrm{I}^{2}$ statistic and publication bias by using a funnel plot.

\section{MAIN RESULTS}

Ten studies met inclusion criteria; nine of these $(n=956)$ provided sufficient information for inclusion in metaanalyses. Methodologically the studies were not strong, with two trials using quasi-randomisation methods, and only one trial having adequate allocation concealment. The trials varied in terms of population included, interventions and usual care provided (see online notes for further details). Psychosocial interventions included peer support or non-directive counselling, while psychological interventions included cognitive behavioural therapy (CBT), interpersonal psychotherapy (IPT), and psychodynamic therapy. When pooled, psychosocial and psychological interventions reduced evidence of depression at the final assessment within the first year after delivery, compared to usual care (nine RCTs, $\mathrm{n}=956$; RR $0.70,95 \%$ CI 0.60 to $0.81 ; \mathrm{I}^{2}=23.5 \%$ ). Psychosocial interventions on their own decreased the likelihood of having evidence of depression when pooled (five RCTs, $\mathrm{n}=506$; RR 0.61, 95\% CI 0.39 to $0.94 ; \mathrm{I}^{2}=55.9 \%$ ) and when analysed by type of intervention (peer support: one RCT, $\mathrm{n}=42$; RR $0.30,95 \% \mathrm{CI}$ 0.10 to 0.92 ; non-directive counselling: four RCTs, $\mathrm{n}=464$; RR $0.67,95 \%$ CI 0.43 to $1.04 ; I^{2}=55.0 \%$ ). Among the psychological interventions, both CBT and IPT reduced evidence of depression compared with usual care, but psychodynamic therapy did not (CBT: five RCTs, $\mathrm{n}=482$; RR $0.72,95 \%$ CI 0.57 to $0.90 ; \mathrm{I}^{2}=0 \%$; IPT: one RCT, $\mathrm{n}=120$; RR $0.80,95 \%$ CI 0.66 to 0.98 ; psychodynamic therapy: one RCT, n =91; RR $0.67,95 \%$ CI 0.33 to 1.37 ).

\section{CONCLUSIONS}

Psychological and psychosocial interventions reduce evidence of depression compared to usual care in the year after delivery in women with postpartum depressive symptoms. Longer term effects are not clear.

\section{ABSTRACTED FROM}

Dennis C-L, Hodnett E. Psychosocial and psychological interventions for treating postpartum depression. Cochrane Database Syst Rev 2007; 4:CD006116.

Correspondence to: Professor Cindy-Lee Dennis, University of Toronto, Lawrence Bloomberg Faculty of Nursing, 155 College Street, Toronto, Ontario, Canada, M5T 1P8; cindylee.dennis@utoronto.ca

Source of funding: No external sources of support reported.

Additional notes are published online only at http://ebmh.bmj.com/content/vol11/ issue 3
$\mathrm{P}$ ostpartum depression (PPD) affects approximately $10-15 \%$ of new mothers $1>20 \%$ in some settings). It has a profoundly negative impact on a mother's experience of parenting and increases the risk of poor attachment and adverse behavioural and emotional outcomes in the offspring. Over the past 10 years several randomised-controlled trials, mainly in the UK, have assessed the short-term efficacy of both antidepressant and non-medical interventions. Most find adequate, if modest, effect sizes for their tested intervention, using reductions in depressive symptomatology as outcome. Few address maternal perception of interventions, their acceptability for women or partners and few measure childhood outcomes beyond the first year. The present review by Dennis and colleagues identified only nine studies of quality for inclusion, and effect sizes for all interventions were moderate.

This review is timely given the reluctance of women to take antidepressants, encouraged by recent evidence of marginal benefit ${ }^{1}$ and of publication bias favouring positive results in antidepressant trials. ${ }^{2}$ The authors conclude that more trials are required with self-help groups, mother-to-mother supports, and research evaluating partner involvement in prevention and recovery. Identified barriers to treatment include stigma, accessibility/transportation, childcare, availability of services and high attrition rates in some groups. Future data should evaluate novel treatments, (including telephone and internet interventions) as well as qualitative data from women, to develop cost-effective services for this complex condition.

In clinical practice local factors remain important. Experience in Manchester suggests a significant effect of violent and abusive relationships in PPD, encouraging the view that mother-to-mother support and sensitive working with families as a whole (including partners) is likely to be very important. In the UK, our unique resource of experienced health visitors and midwives should allow many women to receive assessment and care early in the risk period, but practice must be supported by adequate training and supervision. In future, prioritising equity of access in health may make the internet a good alternative for women waiting for ever-elusive talking therapies.

Kathryn M Abel, MA, MBBS, MRCP, MRCPsych, PhD

Centre for Women's Mental Heath, University of Manchester, Manchester, UK

Competing interests: KA is the PI in Manchester for the multicentre RESPOND study comparing HV delivered active listening and SSRI treatment for PPD in general practice, and has acted as an expert to Wyeth Pharmaceuticals.

1. Kirsch I, Deacon BJ, Huedo-Medina TB, et al. Initial severity and antidepressant benefits: a meta-analysis of data submitted to the food and drug administration. PLoS Med 2008;5:e45.

2. Turner EH, Matthews AM, Linardatos E, et al. Selective publication of antidepressant trials and its influence on apparent efficacy. NEJM 2008;358:25260. 